\title{
Ring-Topology Echo State Networks for ICU Sepsis Classification
}

\author{
Miquel Alfaras ${ }^{1,2}$, Rui Varandas ${ }^{1,3}$, Hugo Gamboa ${ }^{3}$ \\ ${ }^{1}$ PLUX Wireless Biosignals S.A., Lisboa, Portugal \\ ${ }^{2}$ Universitat Jaume I, Castelló de la Plana, Spain \\ ${ }^{3}$ Laboratório de Instrumentação, Engenharia Biomédica e Física da Radiação (LIBPhys-UNL), \\ Departamento de Física, Faculdade de Ciências e Tecnologia da Universidade Nova de Lisboa, \\ Caparica, Portugal
}

\begin{abstract}
Sepsis is a life threatening condition that can be treated if detected early. This paper presents a study of the application of a Ring Topology Echo State Network (ESN) algorithm to a sepsis prediction task based on ICU records. The implemented algorithm is compared with commonly used classifiers and a combination of both approaches. Finally, we address how different causal strategies on filling missing record values affected the final classification performances. Having a dataset with a limited number of time entries per patient, the F1-Score $=14,79 \%$ obtained in the cross-validation stage after optimisation suggests that further research is needed in order for the ESN to capture the temporal dynamics of the problem at hand.
\end{abstract}

\section{Introduction}

Sepsis is defined as "life-threatening organ dysfunction caused by a dysregulated host response to infection" [1]. This condition can be detected using physiological measures, such as, heart rate, temperature and laboratory measures of body fluids analysis. Furthermore, it was identified that any 2 of 3 clinical variables Glasgow Coma Scale score of 13 or less, systolic blood pressure of $100 \mathrm{~mm} \mathrm{Hg}$ or less, and respiratory rate $22 / \mathrm{min}$ or greater offered predictive validity. For patients in the ICU, sepsis prediction is compromised due to the effects of the prescribed treatment. For instance, drugs that help to attenuate the patient symptoms have an impact on physiological measurements (temperature, heart rate, etc.). In turn, mechanical ventilators do not allow to take the respiration rate into account in order to detect sepsis. In [2], a data-driven probabilistic model was found to be able to improve the prediction of sepsis relative to some of commonly used theoretical thresholds.

On the other hand, Machine Learning approaches have been applied in the medical field in contexts such as ar- rhythmia detection [3], death prediction [4], and even death prediction in the presence of sepsis [5]. Hence, we decided to study such techniques for sepsis detection in ICU patients.

Our study was aimed at assessing the application of Echo State Network (ESN) algorithms to predict the occurrence of sepsis in ICU data, with an anticipation of 6 hours. For this goal, we explored the influence of the input parameters and optimised them and compared the results obtained using the ESN with standard classifiers. Finally, we implemented a simple combination of both classic classifiers and ESNs. Additionally, our work addressed the effect that different strategies in the replacement of the missing ICU data had on the results obtained using the ESN.

The trained algorithms can be tested with an unreleased dataset that has never been shown to the classifiers. Their performance is rated according to a utility function that penalises excessively early, late, or false detection and favours timely sepsis detection.

\section{Methods}

The dataset utilised was provided by the organisation of the Physionet Challenge 2019 and consists of physiological, laboratory and demographic measurements of 40,336 patients from the ICU of two different hospitals (20,336 from one and 20,000 from the second). The readings were registered hourly, with missing values appearing as $\mathrm{NaN}$ (not a number). At any point in time, a Sepsis Label indicating the presence of sepsis is provided. Specifically, we count on 40 measurements over 1,552,210 hours, thus making $62,088,400$ values, in which $70 \%$ are NaN. Furthermore, sepsis samples amount to $1.80 \%$ of the total.

\subsection{Preprocessing}

In order to force a $6 \mathrm{~h}$ anticipation in the classification task, the provided data counts already on a $6 \mathrm{~h}$ shift applied to the Sepsis Labels. 
In section 3.4 we study the replacement of the $\mathrm{NaN}$ missing ICU record values by means of:

1. Substitution by zero (constant value);

2. Substitution by the mean value of the patient records;

3. Substitution by a random value assuming a normal distribution with the mean and standard deviation of the original records;

4. Substitution by the value corresponding to the interpolation of the measurements around the missing entries.

The substitutions were applied taking into account the causality of the problem, in which we could only have access to the past measurements until the current time and ignored the future values to simulate the real-time use case.

\subsection{Echo State Network}

The main algorithm chosen for the sepsis classification task consisted in a Ring Topology Echo State Network (ESN). ESNs are a particular subset of recurrent neural networks that leverage Reservoir Computing properties of mapping the input data to a high-dimensional space and simplifying the training given the fact that only output layer weights are trained. Random inner connections are set and kept constant. In a successful implementation, 3 conditions are met: a)The recurrent network exhibits different dynamics for inputs that differ significantly, b)The network provides similar outputs when similar inputs are given, c)The reservoir exhibits a fading memory where temporal dynamics play a relevant role that decays over time.

An Echo State Network consists of an Input layer, a Reservoir and an Output layer.

First, random weights (i.e., mask) map the input to a highly dimensional space, distributing the input along neurons that behave differently so that unique responses are shown for different inputs. Once distributed, inputs need to be converted to a nonlinear space by means of an activation function (such as a sigmoid or hyperbolic tangents $\tanh$ ). The reservoir's internal weights, must recreate a sparsely connected network that preserves the reservoir properties. Finally, the output weights are the only weights subject to training. The training procedure is often guided by the problem of solving the reservoir system presented below.

$$
\hat{S}=E s n \times W
$$

In eq.1, the classification of samples consists in obtaining the sepsis labels $\hat{S}$, i.e. the product of the the reservoir state holding the sample data Esn and the output weights $\mathbf{W}$ that take into account the contribution of the different neurons.

\subsubsection{Topology and ESN formulation}

Recent ESN research drawing upon ring topologies has shown how these networks have successfully been applied to cross-database clinical contexts such as the detection of ventricular heartbeats [6]. We chose to work with the same topology and a zero centred sigmoid nonlinear mapping function $f(x)=\left(1+e^{-x}\right)^{-1}-0.5$. Our mask was continuous, randomly generated, uniformly distributed around 0 and counted on an additive offset per neuron.

Since we counted on a full dataset where the number of samples (s) exceeded 1 million, the ESN reservoir matrix that needs to be numerically inverted has dimensions $E s n=E s n_{s, N}$, which turn to be the computationally limiting factor. Instead of trying to numerically solve a system based on computing the pseudo-inverse matrix of the ESN matrix, we adopted the normal equation formulation presented in [7]. Following eq.1, the reservoir equations are:

$$
\begin{gathered}
E s n^{T} \times S=\left[E s n^{T} E s n\right] W \\
{\left[E s n^{T} E s n\right]^{*} \times\left[E s n^{T} \times S\right]=W}
\end{gathered}
$$

where $S$ represents the known sepsis labels for the training records, Esn is the reservoir matrix holding the information, $W$ are the output weights, $\left({ }^{T}\right)$ stands for matrix transposition and $\left(^{*}\right)$ corresponds to the numerical pseudo-inverse. The advantage of adopting the linear equation formulation lies in the fact that the matrix that needs to be inverted has a constrained dimension of $R \equiv$ $\left[E s n^{T} E s n\right]=R_{N, N}$, significantly less computationally demanding using the LU and Moore-Penrose methods.

The ESN states are represented by:

$$
E s n_{n, t}=f\left(\gamma \text { Input }_{t}+\eta\left(W_{E s n} \times E s n_{n-1, t-1}\right)\right)
$$

where, $E s n_{n, t}$ is the state of neuron $\mathrm{n}$ at time $\mathrm{t}, f$ is the activation function, $\gamma$ is the input scaling factor applied to the masked data Input $_{t}$ and $\eta$ is the memory or leakage parameter controlling the strength between the fixed internal neuron connections $W_{E s n}$. The equation captures the temporal and neighbouring neuron dependencies. In the case of the ring topology, we have a $W_{E s n}$ connection matrix that consists of a square matrix with ones in the subdiagonal.

The optimisation approach was based in the exploration of different regimes for the input scaling and memory parameters $(\gamma, \eta)$. We computed bidimensional grid searches and looked for the best Area Under the Receiver Operating Characteristic Curve (AUC) score by means of a 10-Fold stratified cross-validation strategy, ensuring that no patient is used simultaneously in the training and test subsets. 


\section{Results}

In this section we present a comparison of sepsis classification performances of the ESN and other classifiers. Regarding the problem that the absence of values posed, we first adopted the strategy of substituting the missing $\mathrm{NaN}$ values by zeros and proceeded with the exploration of other approaches reported below. All performances are reported in the form of percentages $\%$. We used the AUC to guide our parameter optimisation, and proceeded to apply the optimal threshold and compute the corresponding F1score, Precision (Pr), Recall (Re) and Accuracy (ACC) for the highest AUC point $(\eta, \gamma)$. We checked for robustness of the ESN and input mask dependence by means of ensembles (5-10 networks) that yield 2-3\% increases in AUC. When reporting performances we use F1-score, given that it combines Pr and $\mathrm{Re}$, which are the most important metrics in extremely imbalanced data sets like ours.

\subsection{Echo State Network Parameters}

In the case of the ESN, we started assessing the influence of the amount of neurons employed (N). The reservoir size is a key element for a reservoir computer to be able to either find similarities or tell samples apart. Since its use is motivated by a high dimensional nonlinear mapping, the number of neurons must be greater than the number of features provided at any given point. In order to find out an optimal regime for our network to work, we used the first data subset of 5000 patients and conducted a grid search on several reservoir sizes (of $\mathrm{N}$ neurons) scanning over the same ranges of memory and input scaling parameters $(\eta, \gamma \in[0 ; 10])$.

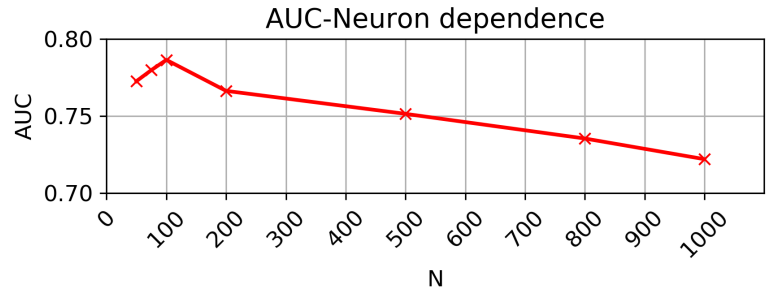

Figure 1. Study of the AUC-Neuron dependence

Fig. 1 shows that $\mathrm{N}=100$ provided the highest AUC values. Once the $\mathrm{N}$ size was set, we conducted grid searches in the $(\gamma, \eta)$ parameter space using the whole dataset. Fig.2 shows a representation of the resulting parameter map.

Table 1 shows the results for the given optimal parameters in terms of AUC $(\mathrm{N}=100, \gamma \leq 0.001, \eta \in[0.1 ; 2.5])$. The trained ESN received a positive utility score of 0.206 in the official submission of the Physionet Challenge 2019.

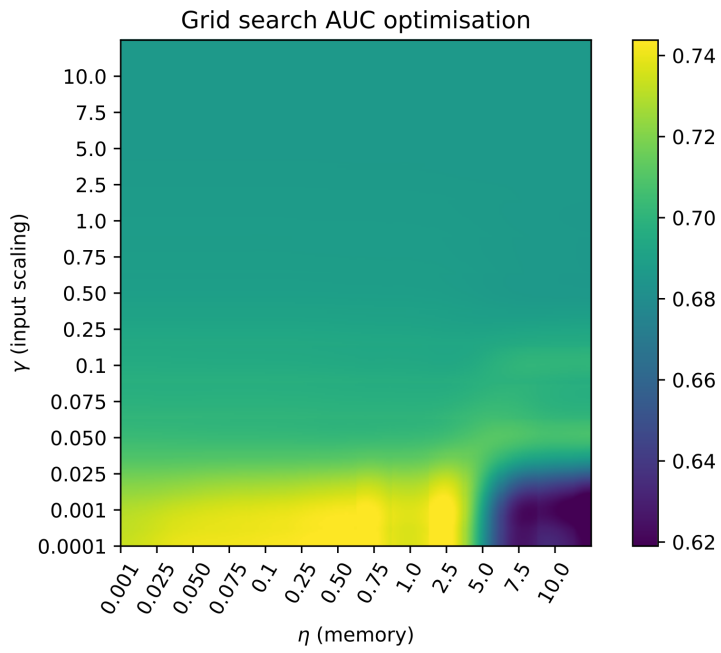

Figure 2. AUC - Grid search ESN parameter optimisation

Table 1. Results obtained using the AUC-optimised ESN.

\begin{tabular}{llll}
\hline \hline Accuracy & F1-Score & Recall & AUC \\
\hline 95.97 & 14.79 & 19.44 & 74.38 \\
\hline \hline
\end{tabular}

\subsection{Classifier comparison}

In this work we used four different classifiers available in the scikit-learn Python package [8]: Decision Trees (DT), Gaussian Naive-Bayes (GNB), Random Forest (RF) and Gradient Boosting (GB) classifier. Here, we wanted to compare the performance of our ESN in relation to the performance obtained using different classifiers (Table 2).

Table 2. Results of different classifiers.

\begin{tabular}{lllcc}
\hline \hline Classifier & Accuracy & F1-Score & Recall & AUC \\
\hline DT & 95.77 & 8.78 & 11.32 & 54.33 \\
GNB & 81.00 & 7.28 & 41.50 & 66.45 \\
RF & 95.28 & 16.80 & 26.51 & 77.21 \\
GB & 95.46 & 18.29 & 28.25 & 80.14 \\
\hline \hline
\end{tabular}

These results indicate that some classifiers may be better suited for this specific problem than the application of ESN, namely, the RF and GB classifiers.

\subsection{Combination of ESN and classifiers}

The combination of the ESN with other classifiers could possibly increase the performance of both methods. The combination was made by implementing the network described in section 2 and providing the resulting neuron states as the input for the classifiers (see results in Table 3). 
Table 3. ESN-classifier combination results.

\begin{tabular}{lllcc}
\hline \hline Combination & Accuracy & F1-Score & Recall & AUC \\
\hline ESN + DT & 95.95 & 6.75 & 8.15 & 52.86 \\
ESN + GNB & 88.94 & 6.09 & 19.94 & 61.96 \\
ESN + RF & 95.00 & 14.44 & 23.46 & 74.87 \\
ESN + GB & 95.14 & 15.34 & 24.51 & 72.49 \\
\hline \hline
\end{tabular}

The combined classifications suggest that despite achieving values in some cases comparable to those of the ESN (e.g. ESN+RF, ESN+GB), classifier performances using the network as input are worsened when compared to their raw input counterparts (see Tables 3 and 2).

\subsection{Causal NaN Substitution Influence}

In an early stage, we addressed noncausal scenarios where a relevant cross-hospital classification (AUC > $80 \%$ ) was achieved, enhanced by a minmax scaling and $\mathrm{NaN}$ substitution. Given the number of missing NaN values, we saw how strategies to replace them could help algorithms better capture the patterns in the data. In this section we address different ways of causally substituting those missing values, ranging from the simplest constant value substitution (0), to more complex methods such as neighbouring value interpolation. The results are presented in Table 4, in which each number refers to the strategy described in section 2.1

Table 4. Influence of the NaN substitution on the ESN. Each number corresponds to the description on section 2.1 .

\begin{tabular}{lllcc}
\hline \hline Substitution & Accuracy & F1-Score & Recall & AUC \\
\hline (1) Const. & 95.97 & 14.79 & 19.44 & 74.38 \\
(2) Mean & 96.20 & 13.91 & 17.08 & 75.94 \\
(3) Norm. & 92.94 & 15.20 & 35.22 & 75.64 \\
(4) Interp. & 95.30 & 15.49 & 23.95 & 75.90 \\
\hline \hline
\end{tabular}

\section{Conclusions}

We have successfully programmed, trained, optimised and tested a Python implementation of a Ring Topology ESN for a Sepsis prediction task. While the sepsis prediction applied to an unseen dataset provided a positive rated utility score, the low performance achieved by the ESN relative to other classifiers in the cross-validation stage suggests that further research is needed in order for the ESN to capture the temporal dynamics of the problem at hand. Having a highly imbalanced dataset with a limited number of time entries per patient, we hypothesise that research into data augmentation approaches maintaining a coher- ence in how regularly the data is fed to the ESN in a causal use case scenario could yield to better sepsis prediction.

Although enhanced by the use of other classifiers drawing upon the mapped reservoir states, we can conclude that no implementation based on our ESN outperforms that of commonly used classifiers so far. Finally, while the $\mathrm{NaN}$ replacement strategy plays a key role in non-causal use case scenarios, no significant F1-Score improvements were observed in the causal classification task.

\section{Acknowledgements}

This work was supported by Marie Skłodowska Curie Actions ITN AffecTech (ERC H2020 Project ID: 722022).

\section{References}

[1] Singer M, Deutschman CS, Seymour CW, Shankar-Hari M, Annane D, Bauer M, Bellomo R, Bernard GR, Chiche JD, Coopersmith $\mathrm{CM}$, et al. The third international consensus definitions for sepsis and septic shock (sepsis-3). JAMA 2016;315(8):801-810.

[2] Tsoukalas A, Albertson T, Tagkopoulos I. From data to optimal decision making: a data-driven, probabilistic machine learning approach to decision support for patients with sepsis. JMIR medical informatics 2015;3(1):e11.

[3] Eerikäinen LM, Vanschoren J, Rooijakkers MJ, Vullings R, Aarts RM. Decreasing the false alarm rate of arrhythmias in intensive care using a machine learning approach. In Computing in Cardiology Conference. IEEE, 2015; 293-296.

[4] Johnson AE, Kramer AA, Clifford GD. Data preprocessing and mortality prediction: The physionet/cinc 2012 challenge revisited. In Computing in Cardiology. IEEE, 2014; 157160.

[5] Taylor RA, Pare JR, Venkatesh AK, Mowafi H, Melnick ER, Fleischman W, Hall MK. Prediction of in-hospital mortality in emergency department patients with sepsis: a local big data-driven, machine learning approach. Academic emergency medicine 2016;23(3):269-278.

[6] Alfaras M, Soriano MC, Ortín S. A fast machine learning model for ECG-based heartbeat classification and arrhythmia detection. Frontiers in Physics 2019;7:103.

[7] Lukoševičius M. A practical guide to applying echo state networks. In Neural networks: Tricks of the trade. Springer, 2012; 659-686.

[8] Pedregosa F, Varoquaux G, Gramfort A, Michel V, Thirion B, Grisel O, Blondel M, Prettenhofer P, Weiss R, Dubourg V, Vanderplas J, Passos A, Cournapeau D, Brucher M, Perrot M, Duchesnay E. Scikit-learn: Machine learning in Python. Journal of Machine Learning Research 2011;12:2825-2830.

Address for correspondence:

Miquel Alfaras - PLUX S.A.

Avenida 5 de Outubro 70, Lisboa (Portugal)

malfaras@plux.info 